\title{
On the Hidden Beauty of Trigonometric Functions
}

\author{
Jiří Stávek ${ }^{1}$ \\ ${ }^{1}$ Bazovského 1228, 16300 Prague, Czech republic \\ Correspondence: Jiří Stávek, Bazovského 1228, 16300 Prague, Czech republic. E-mail: stavek.jiri@seznam.cz
}

Received: February 26, 2017

Accepted: March 10, $2017 \quad$ Online Published: March 17, 2017

doi:10.5539/apr.v9n2p57

URL: https://doi.org/10.5539/apr.v9n2p57

\begin{abstract}
In the unit circle with radius $\mathrm{R}=\mathrm{E}_{0}=\mathrm{mc}^{2}=1$ we have defined the trigonometric function $\cos (\theta)=\mathrm{v} / \mathrm{c}$. The known trigonometric functions revealed the hidden relationships between sensible energy, latent energy, sensible momentum and latent momentum of the moving object, and the absorbed momentum from outside and the available momentum in the outside of the moving object. We present the trigonometric concept inspired by the old Babylonian clay tablet IM 55357 and based on the knowledge of the School of Athens (the fresco of Raphael) and the work of many generations of the Masters of trigonometry. The concept of the Divided Line of Plato can be now quantitatively tested. For the experimental analysis of this concept we propose to study in details the very well known beta decay of RaE to determine the sensible and latent energy (heat) of those beta particles and the sensible and latent energy of the remaining nucleus. The longitudinal momentum and the transverse (latent) momentum can be studied on the effects of the slow neutrons. The longitudinal momentum and the transverse momentum of photons can be manipulated in a convenient medium in order to prepare slow photons. The photoormi effect might improve the efficiency of the light-to-electricity conversion and the efficiency of the lightto-heat conversion.

Keywords: Trigonometric function $\cos (\theta)=\mathrm{v} / \mathrm{c}$, sensible energy, latent energy, sensible momentum, latent momentum, absorption of momentum from the outside, beta decay of RaE, slow neutrons, slow photons, photoormi effect
\end{abstract}

\section{Introduction}

Each mathematical language describing the quantities of motion (energy and momentum) reveals some parts of that complex behavior while the other parts are hidden and cannot be discovered by that mathematical language (Kuhn, 1962). One of those mathematical languages - trigonometry - was developed by many generations during a long period of time on several places. The historical overview of the development of trigonometry can be found in the works of Von Braunmühl (1900), Datta and Singh (1983), Matviesvkaya (1990), Maor (1998), and Van Brummelen $(2009,2013)$.

The trigonometric functions played always a very important role in the development of physics and astronomy: Hipparchus and Claudius Ptolemy (chord), Nicolaus Copernicus (half chord = sine), Johannes Kepler (sine, versine, tangent), Galileo (exsecant, tangent), Newton (sagitta, tangent), Leibniz and Huygens (cosecant - sine = vis viva), Einstein (cosecant, excosecant), Planck (cotangent). (E.g., Hawking, 2002).

We were inspired by Leibniz - "Sometimes in physics, to move forwards, it is helpful to look backwards". The first inspiration we have found on the old Babylonian clay tablet IM 55357 (Friberg, 2007). The second source of inspiration came to us from the School of Athens (see the fresco of Raphael). There are depicted many Masters who founded a strong base for further development of their concepts. E.g., the Divided Line of Plato - a measure of the human knowledge can be now trigonometrically quantitatively tested.

If we define in the unit circle with radius $\mathrm{R}=\mathrm{E}_{0}=\mathrm{mc}^{2}=1$ the trigonometric function $\cos (\theta)=\mathrm{v} / \mathrm{c}$, then we will get automatically many relationships between sensible and latent energy, sensible and latent momentum of the moving object, and the absorbed momentum from the outside and the available momentum in the outside. This trigonometric approach can be experimentally tested during the beta decay of $\mathrm{RaE}$ to determine the sensible and latent energy (heat) of those beta particles and the sensible and latent energy of the remaining nucleus. The longitudinal momentum and the transverse (latent) momentum can be studied on the effects of the slow neutrons. The photoormi effect (the manipulation of the momentum of slow photons) might improve the efficiency of the light-to-electricity conversion and the efficiency of the light-to-heat conversion. 
There are three concepts of mass: for the fermion mass $2 / 3$ of researchers use the "modern" view $\mathrm{E}_{0}=\mathrm{mc}^{2}$ (the fermion mass is not velocity dependent), $1 / 3$ of researchers use the "archaic" concept $E=\mathrm{mc}^{2}$ (the mass of fermions depends on the velocity). For photon mass almost all researchers use the result coming from the relativistic equations: photon mass at $\mathrm{c}$ is $\mathrm{m}=\mathrm{m}$, while photon mass at $0 \mathrm{~km} / \mathrm{s}$ equals $\mathrm{m}=0$. This rest mass postulate for photons is the necessary condition for the validity of the relativistic theory. For the detailed discussion see the great book of Okun (2009).

\section{Trigonometric Functions in the Unit Circle with Radius $R=E_{0}=m c^{2}=1$ and $\cos (\theta)=v / c$}

Figure 1 shows trigonometric functions that will be used in this trigonometric model.

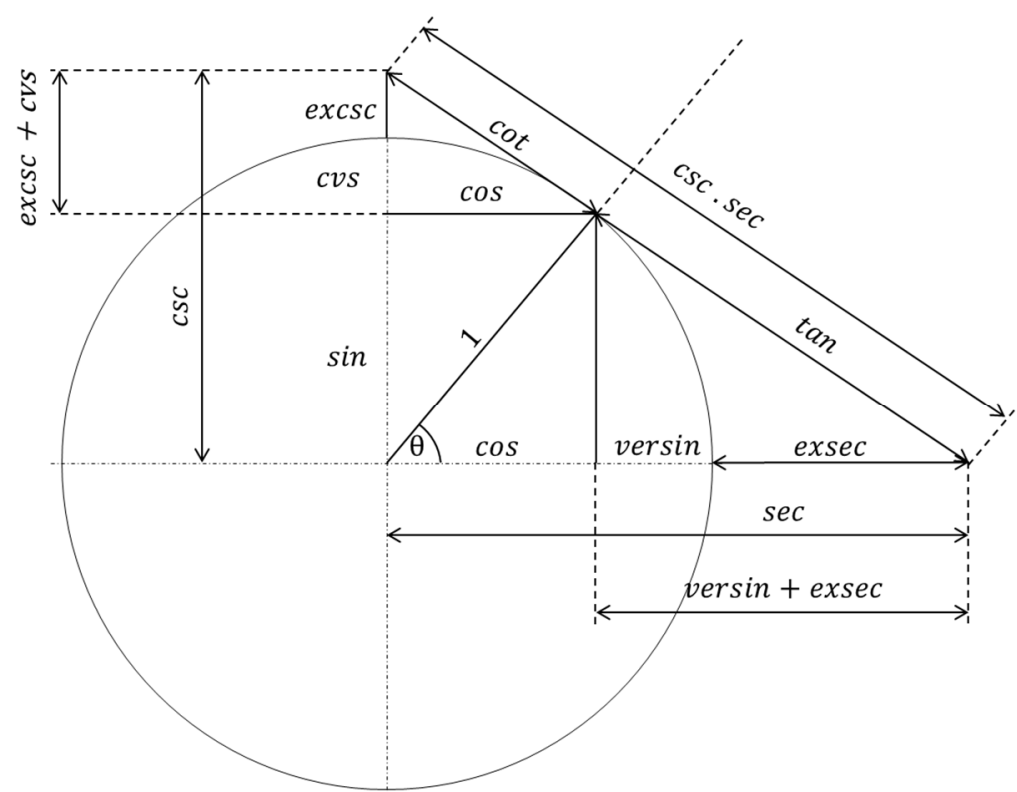

Figure 1. Trigonometric functions for the angle $\theta$

Table 1. gives the values of those used trigonometric functions for $\cos (\theta)=\mathrm{v} / \mathrm{c}$

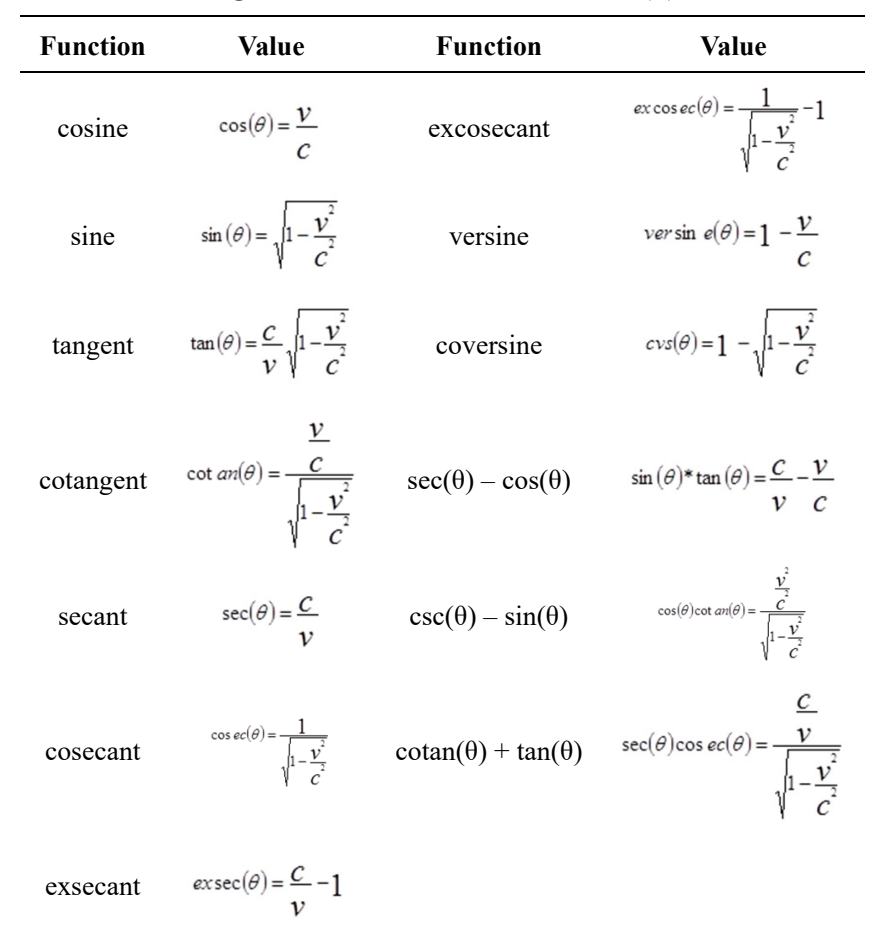




\section{The Divided Line of Plato}

Plato developed the original philosophical concept - the Divided Line of Plato - that could be used for the quantitative determination of human knowledge.

We were inspired by the old Babylonian clay tablet IM 55357 where we have described the lengths of lines using the trigonometric functions. We have found that ratios of those lengths fulfill conditions proposed by Plato in his concept as it is shown in Figure 2.

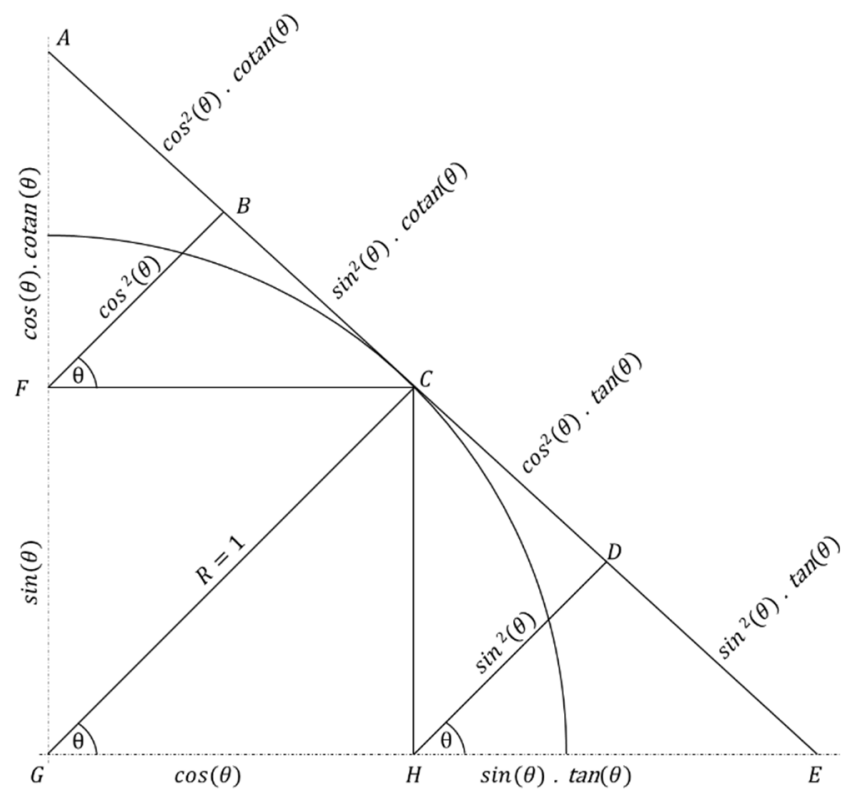

Figure 2. The Divided Line of Plato ABCDE

Based on the properties of similar triangles in Figure 2 we can determine ratios to be equal $\operatorname{cotan}^{2}(\theta)$ :

$$
\cot \operatorname{cn}^{2}(\theta)=\frac{A B}{B C}=\frac{C D}{D E}=\frac{A C}{C E}=\frac{A F}{F G}=\frac{G H}{H E}=\frac{A F+F C}{C H+H E}=\frac{B F}{D H}
$$

For high values of $\cos (\theta)=\mathrm{v} / \mathrm{c}$ we can say that our knowledge is impressive - this is fulfilled for individual electrons and protons. However, for macroobjects with number of particles on the level of $6.022 * 10^{23}$ we can achieve the values of $\cos (\theta) \approx 10^{-4}$ - this means that our actual knowledge measured by the Plato's concept is still rather limited.

This concept of the Divided Line of Plato reveals a new possibility how to increase the quantity of our knowledge. We might develop techniques for the manipulation with momentum of individual particles and to synchronize their motion.

\section{Hidden Energy and Hidden Momentum of Fermions - how to Divide the Cosecant?}

The rest energy $\mathrm{E}_{0}$ was the greatest discovery of the $20^{\text {th }}$ century. Einstein found that massive body has a huge hidden energy $\mathrm{E}_{0}=\mathrm{mc}^{2}$. We will avoid the use of the "archaic" concept of the relativistic mass and will use the "modern" view that mass is not velocity dependent. For the detailed discussion see the great book of Okun (2009).

Several generations of experimentalists clearly demonstrated and verified that the total energy of the moving objects with fermions can be described using the cosecant:

$$
E=E_{0}{ }^{*} \csc (\theta)=m c^{2} \frac{1}{\sqrt{1-\frac{v^{2}}{c^{2}}}}
$$

In order to describe the kinetic energy of fermions Albert Einstein devided the cosecant into the excosecant and radius: 


$$
E=E_{0} * \csc (\theta)=m c^{2}\left[\left(\frac{1}{\sqrt{1-\frac{v^{2}}{c^{2}}}-1}\right)+1\right]
$$

In our proposed trigonometric model we were inspired by the Divided Line of Plato and by the vis viva concept of Leibniz and Huygens and divided the cosecant into two parts - the first part should describe the sensible energy of fermions and the second part the latent energy of fermions that could be revealed as heat (transverse vibrations):

$$
\begin{gathered}
E=E_{0}{ }^{*} \csc (\theta)=m c^{2}\left[\left(\frac{1}{\sin (\theta)}-\sin (\theta)\right)+\sin (\theta)\right] \\
E=E_{0}{ }^{*} \csc (\theta)=m c^{2}\left(\frac{\frac{v^{2}}{c^{2}}}{\sqrt{1-\frac{v^{2}}{c^{2}}}}+\sqrt{1-\frac{v^{2}}{c^{2}}}\right)
\end{gathered}
$$

Figure 3 shows a right triangle with two legs: cosecant and secant, and the hypotenuse equals to the product of secant*cosecant. If we split those two legs and hypotenuse into parts that obey the condition of the Divided Line of Plato then we might get the expressions for the sensible energy, latent energy, sensible momentum, latent momentum, and the absorbed momentum from the outside that caused the motion and the available momentum in the outside that could be used for the further addition into the moving system.

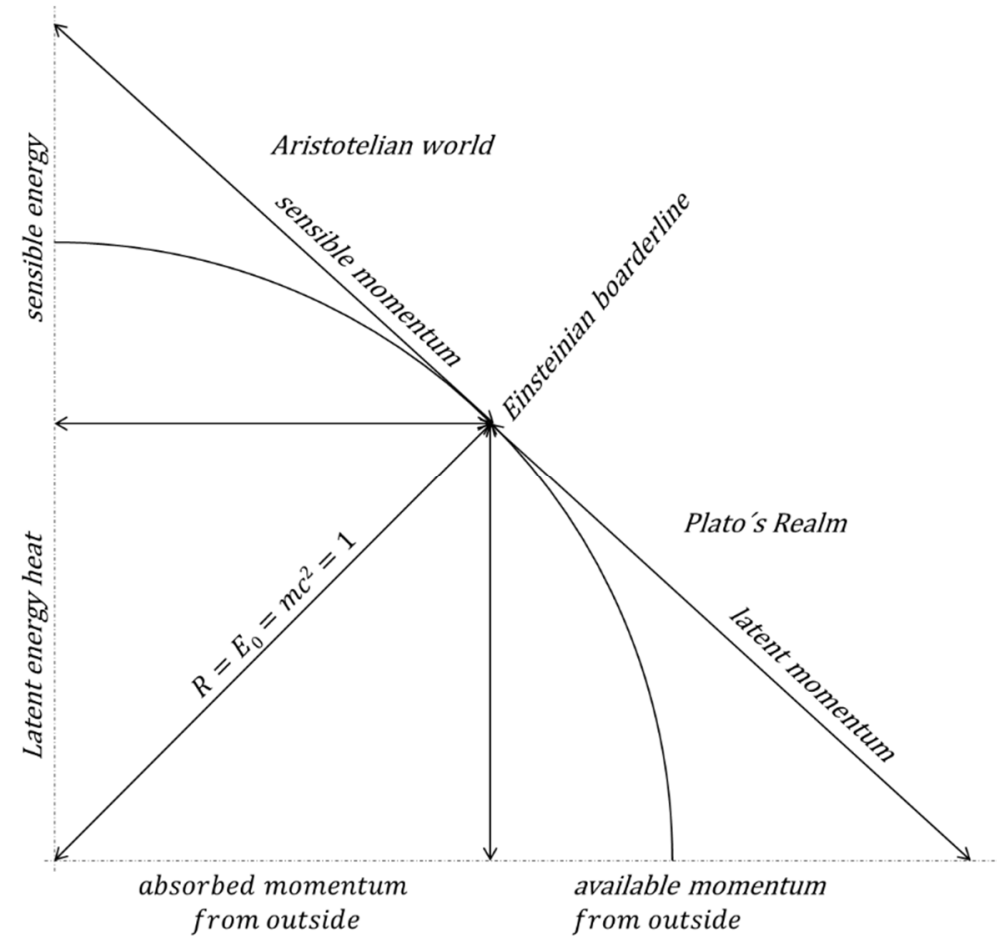

Figure 3. The division of cosecant, secant and the hypotenuse ( $\left.\mathrm{csc}^{*} \mathrm{sec}\right)$

Figure 4 shows the quantitative values of sensitive and latent transverse energies, and the sensitive and latent transverse momentums that could be experimentally tested for the case of slow neutrons. Enrico Fermi discovered on October 22, 1934 that the slow neutrons have much higher efficiency in some reactions (Bruzzaniti, 2010). This efficiency is usually described by the barn units $\left(10^{-28} \mathrm{~m}^{2}\right)$ and interpreted as the "effective capture" of slow neutrons. It is very well known that this "capture" of slow neutrons depends on their velocity as $\approx 1 / \mathrm{v}$ but the precise formula is not known yet (e.g., J. Byrne in 2011). We propose to explain the efficiency of slow neutrons in these reactions by their transverse momentum - the tangent formula. 


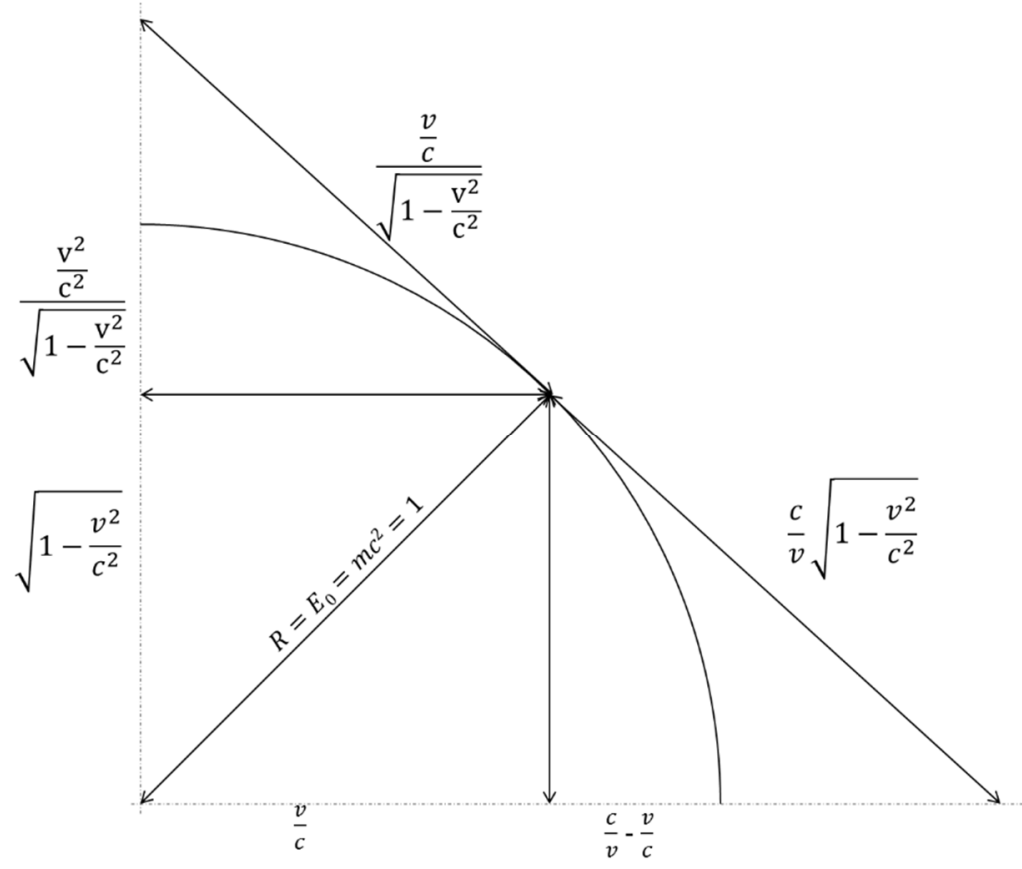

Figure 4. The quatities of motion for fermions

\section{Beta decay of RaE}

It is very well known that the beta decay process is full of surprises and subtleties (e.g., Crane, 1948, Wu, 1966, Franklin, 2000, Jensen et al., 2000). There were observed several anomalies that could not be described by the relativistic formulae: the continuous spectrum of beta particle energies, the missing energy and the missing momentum. In order to save the relativistic description using the excosecant (kinetic energy) and the cotangent (sensible momentum) Wolfgang Pauli in 1930 proposed to postulate a new particle carrying away those missing energy and momentum. Later Enrico Fermi in 1933 developed his theory based on the neutrino creation that carries away the "missing energy" and "missing momentum".

We propose to re-open the discussion about the analysis of the experimental data found for the case of the beta decay of RaE:

$$
B i_{33}^{210} \rightarrow P O_{34}^{210}+e_{-1}^{0}
$$

The value $\mathrm{Q}=1.161 \mathrm{MeV}$ (the difference between the initial and final nuclear mass energies) for this case could not be fully experimentally observed. There were done two types of experiments. In the first type the mean sensitive energy of moving beta particles was found to be $0.404 \pm 0.020 \mathrm{MeV}$ and in the second calorimetric studies the mean heat effect per one beta decay was found to be $0.337 \pm 0.020 \mathrm{MeV}$. Both Pauli and Fermi these two experimental values equaled as the same experimental value with the same physical interpretation.

In our trigonometric model we propose to interpret these two values as two different experimentally observed effects: the sensitive energy (vis viva) $=$ excosecant plus coversine, and the latent energy $=$ sine that should be revealed as heat. The remaining missing energy from the beta decay should be hidden in the formed polonium atom as its sensitive energy and transverse energy. Because the polonium mass is much higher then the beta particle mass, then the resulting effect will be on the level of units of electronvolts. The missing momentum should be revealed as the transverse momentum of formed beta particles and experimentally could be studied on the slow beta particles. Heat effect of slow beta particles should be significantly higher then heat effect of fast beta particles.

The energy and momentum of formed beta particles can be quantitatively studied as:

$$
\frac{E}{m_{e} c^{2}}=\csc (\theta)=\left(\frac{\frac{v^{2}}{c^{2}}}{\sqrt{1-\frac{v^{2}}{c^{2}}}}+\sqrt{1-\frac{v^{2}}{c^{2}}}\right)
$$




$$
\frac{P}{m_{i} c}=\cot a n(\theta)+\tan (\theta)=\left(\frac{\frac{v}{c}}{\sqrt{1-\frac{v^{2}}{c^{2}}}}+\frac{c}{v} \sqrt{1-\frac{v^{2}}{c^{2}}}\right)
$$

The experimentally observed and predicted parameters of beta particles and the remaining polonium atom in $\mathrm{RaE}$ decay are summarized in Figure 5.

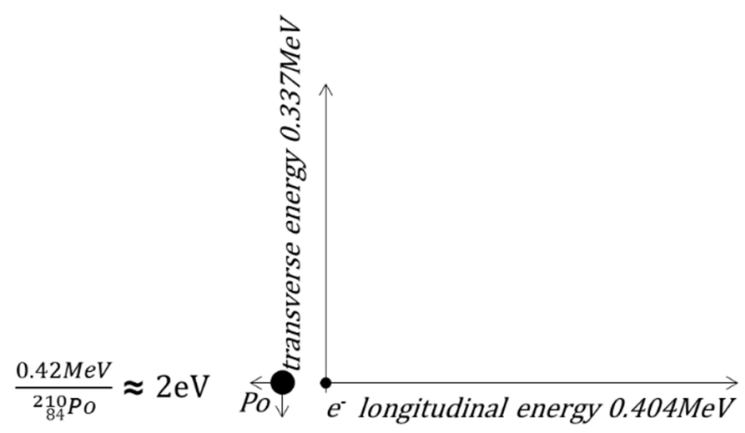

Figure 5. RaE beta decay

\section{Photon Energy and Momentum in a Medium}

For the description of photon energy $\mathrm{E}$ in a medium, where $\mathrm{v}<\mathrm{c}$, we can use coversin $(\theta)$ for the longitudinal energy of that photon and $\sin (\theta)$ for the transverse energy (heat) of that photon:

$$
\frac{E_{0}}{m c^{2}}=c v s(\theta)+\sin (\theta)=\left(1-\sqrt{1-\frac{v^{2}}{c^{2}}}\right)+\sqrt{1-\frac{v^{2}}{c^{2}}}
$$

For the description of the photon momentum $\mathrm{P}$ in a medium the trigonometric function $\cos (\theta)$ determines the longitudinal momentum in that given medium, and the versin $(\theta)$ evaluates the transverse momentum of that photon:

$$
\frac{P}{m c}=\cos (\theta)+v e r \sin (\theta)=\frac{v}{c}+\left(1-\frac{v}{c}\right)
$$

This scenario is depicted in Figure 6. The application of proposed trigonometric functions for the properties of photons in a medium enables to keep the "modern" concept of the mass that should not depend on the velocity of that particle (Okun, 2009). Until now, we have two limits for the mass of photons: one for $\mathrm{c}(\mathrm{m}=\mathrm{m})$, and the second one for the rest photon $(\mathrm{m}=0)$ in order to fulfill conditions of the relativistic formulae.

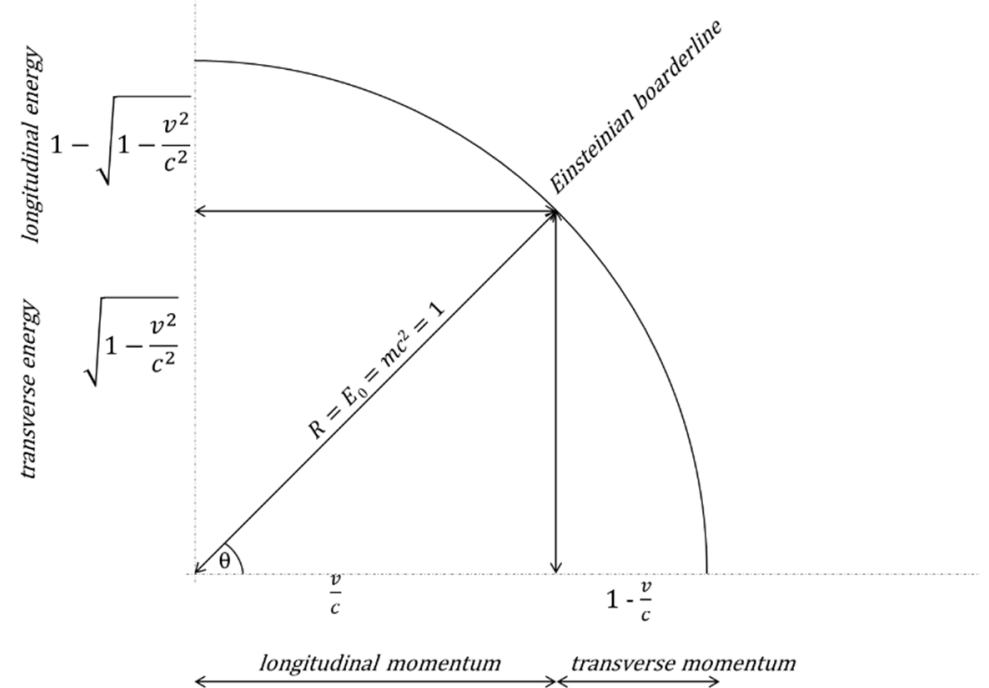

Figure 6. The quantities of motion for photons 
For the speed of light in a medium the refractive index $\mathrm{n}=\mathrm{c} / \mathrm{v}$ stands for the ratio between $\mathrm{c}$ and the speed $\mathrm{v}$ at which light travels in a transparent material. We can manipulate with the speed of photons in different media (air, liquids, metals, crystals, suspensions, coatings, etc.) and thus prepare slow photons with the desired speed. In the next step these slow photons might illuminate a selected target (as e.g. a convenient semiconductor) and promote two conversions: a) light-to-electricity conversion by a release of an electron, and b) light-to-heat conversion through vibration of atoms or molecules.

We can study the effects of slow photons using these two relations:

$$
\begin{aligned}
& \frac{E_{0}}{m c^{2}}=\operatorname{cvs}(\theta)+\sin (\theta)=\left(1-\sqrt{1-\frac{1}{n^{2}}}\right)+\sqrt{1-\frac{1}{n^{2}}} \\
& \frac{P}{m c}=\cos (\theta)+v e r \sin (\theta)=\frac{1}{n}+\left(1-\frac{1}{n}\right)
\end{aligned}
$$

\section{Photoormi Effect - Manipulation with Photon Momentum in a Medium}

It was proposed by Albert Einstein and experimentally very well documented that the photoelectric effect depends strongly on the total momentum of photons while irradiating surface of metals by those photons.

We propose to study in details the photoelectric effect with slow photons in order to reveal if this photoelectric effect is influenced by the longitudinal momentum or by the transverse momentum of slow photons. We propose to call this technique with the tailor-made manipulation of momentum of photons as the Photoormi Effect. The Greek word $о \rho \mu \dot{\eta}$ - ormi is the expression for momentum. This process could be an analogy to Fermi's method with slow neutrons. However, in this case we have been working with energies on the level of units of electronvolts if we manipulate with the photons with wavelengths between $380-760 \mathrm{~nm}$.

It will be interesting to study the efficiency limit of slow photons and compared the obtained efficiency limit with the Shockley-Quisser limit. Another interesting goal might be to achieve a release of several electrons from the action of one slow photon.

This knowledge might improve our strategy in the development of new materials used for the light-to-electricity conversion or for the light-to-heat conversion (e.g., Reinders et al., 2017).

This photoormi effect might quantitatively reveal two faces of the photons that were always described by old Masters as light and heat.

\section{Conclusions}

1) In the unit circle with radius $\mathrm{R}=\mathrm{E}_{0}=\mathrm{mc}^{2}=1$ we have defined the trigonometric function $\cos (\theta)=\mathrm{v} / \mathrm{c}$.

2) The concept of the Divided Line of Plato was used as the guiding principle in the division of cosecant, secant and the hypotenuse of this right triangle (secant*cosecant).

3) The known trigonometric functions revealed the hidden relationships between sensible energy, latent energy, sensible momentum and latent momentum of the moving object, and the absorbed momentum from outside and the available momentum in the outside of the moving object.

4) This trigonometric concept can be experimentally evaluated in the beta decay of RaE.

5) This trigonometric concept can be experimentally evaluated in the processes with slow neutrons.

6) The trigonometric functions for the longitudinal energy and transverse energy of photons in a medium were proposed $-\operatorname{cover} \sin (\theta)$ and $\sin (\theta)$. The trigonometric function $\cos (\theta)$ for the longitudinal momentum of photon in a medium was used. The trigonometric function versin $(\theta)$ describes the transverse momentum of slow photons.

7) The photoormi method was proposed for the manipulation with momentum of slow photons in order to increase the efficiency of the light-to-electricity conversion and the efficiency of the light-to-heat conversion.

\section{Acknowledgments}

This work was supported by the JP\&FŠ Agency (Contract Number 25g/1963), by the VZ\&MŠ Agency (Contract Number 16000/1989) and by the GMS Agency (Contract Number 69110/1992). We have found the valuable support on the web site www.wolframalpha.com with the corrections of used formulae.

\section{References}

Bruzzaniti, G. (2010). Enrico Fermi. The Obedient Genius. Springer, New York. http://dx.doi.org/10.1007/978-14939-3533-8 
Byrne, J. (2011). Neutrons, Nuclei \&Matter. An Exploration of Physics of Slow Neutrons. Philadelphia: Institute of Physics Pub.

Crane, R. H. (1948). The Energy and Momentum Relations in the Beta-Decay, and the Search for the Neutrino. Rev. Mod. Physics, 20, 278-295. http://doi.org/10.1103/RevModPhys.20.278

Datta, B., \& Singh, A. N. (1983). Hindu Trigonometry. Indian Journal of History of Science, 18, 39-108. Retrieved from www.insa.nic.in/writereaddata/.../IJHS/Vol18_1_5_BDatta.pdf

Franklin, A. (2000). Are there Really Neutrinos? An Evidential History. Perseus Books, Cambridge.

Friberg,. J. (2007). Amazing Traces of a Babylonian Origin in Greek Mathematics. World Scientific Publishing, Sinagapore. Retrieved from www.worldscientific.com/doi/pdf/10.../9789812708847_fmatter

Hawking, S. (Ed.) (2002). On the Shoulders of Giants. The Great Works of Physics and Astronomy. Running Press, Philadelphia.

Jensen, C., Aaserud, F., Kragh, H., Rüdinger, E., \& Stuewer, R. H. (2000). Controversy and Consensus: Nuclear Beta Decay 1911:1934. Birkhäuser Verlag, Basel. http://dx.doi.org/10.1007/978-3-0348-8444-0

Kuhn, S. T. (1962). The Structure of Scientific Revolutions. Chicago: University of Chicago Press.

Maor, E. (1998). Trigonometric Delights. Princeton: Princeton University Press. Retrieved from http://freebooksdl.com/346564-trigonometric-delights-8.html/

Matvievskaya, G. P. (1990). An outline of History of Trigonometry. Tashkent: Fan. Retrieved from $\mathrm{http}: / / \mathrm{www} . y e l l o w p a g e s . u z / c o m p a n y / 11$

Okun, L. B. (2009). Energy and Mass in Relativity Theory. World Scientific Publishing, Singapore. http://dx.doi.org/10.1142/6833

Pauli, W. (1930). Letter about the new particle. Retrieved from http://microboone-docdb.fnal.gov/cgibin/RetrieveFile?docid=953; filename=pauli\%20letter1930.pdf

Reinders, A., Verlinden, P., van Sark, W., \& Freundlich, A. (2017). Photovoltaic Solar Energy: From Fundamentals to Applications. John Wiley \& Sons, Chichester. http://dx.doi.org/10.1002/9781118927496.ch1

Van Brummelen, G. (2009). The Mathematics of the Heavens and the Earth (The early history of trigonometry). Princeton: Princeton University Press.

Van Brummelen. G. (2013). Heavenly Mathematics (The forgotten art of spherical trigonometry). Princeton, Princeton University Press.

Von Braunmühl, A. (1900). Vorlesungen über Geschichte der Trigonometrie. Leipzig, Teubner. Retrieved from https://archive.org/details/vorlesungenber00brauuoft

Wu, S., \& Moszkowski, S. A. (1966). Beta Decay. Interscience Publishers, New York.

\section{Copyrights}

Copyright for this article is retained by the author(s), with first publication rights granted to the journal.

This is an open-access article distributed under the terms and conditions of the Creative Commons Attribution license (http://creativecommons.org/licenses/by/4.0/). 